\title{
A Double Nellix and Chimney Covered Stents: Challenging Treatment of Pararenal Aortic Aneurysm
}

Vascular and Endovascular Surgery I-6

\author{
Nazzareno Stella, MD', Roberta Ficarelli, MD', Raffaele Dito, MD', \\ Domitilla Brancadoro, MD², Michele Rossi, MD ${ }^{3}$, \\ and Maurizio Taurino, MD'
}

\begin{abstract}
A 77-year-old male patient presented with a symptomatic, 66-mm pararenal aortic aneurysm. The patient was classified as unsuitable for open surgery due to significant comorbidities. Fenestrated or branched endografts were contraindicated due to the poor iliac access $(6 \mathrm{~mm}$ diameter). A double Nellix with chimney endovascular aneurysm sealing (ChEVAS) technique was selected to exclude the pararenal aortic aneurysm and to preserve renal arteries and the superior mesenteric artery. Technical preplanning considered the ideal proximal landing zone to be close to the origin of the almost occluded celiac trunk and the distal common iliac arteries as the ideal distal landing zone. The total length of the aorta to cover was estimated as $>180 \mathrm{~mm}$, requiring 2 aortic EVAS systems, bilaterally overlapped. Technical success was achieved, and the patient was discharged on postoperative day 8 in good general condition. Successful aneurysm exclusion and target vessel patency without endoleak or stent-graft kinking or migration were confirmed at angio-computed tomography at 6 months.
\end{abstract}

\section{Keywords}

aortic aneurysm, EVAS, chimney technique, totally percutaneous approach

\section{Introduction}

The introduction of fenestrated and branched endografts for endovascular aneurysm repair (f/bEVAR) allowed the treatment of ever more complex aortic anatomies, including juxtarenal and suprarenal aortic aneurysms. Early and midterm results are promising. ${ }^{1,2}$ Although $\mathrm{f} / \mathrm{bEVAR}$ has extended the applicability of EVAR, the technique is challenging and has been associated with short- and long-term complications, including endoleaks, endograft migration, kinking, and stent fractures. $^{3-5}$ Moreover, f/bEVAR requires a custommanufactured endograft, based on individual patient anatomies, consequently precluding its use in acute settings.

The chimney technique has been utilized in patients with juxtarenal and suprarenal aortic aneurysms, deemed unfit for open surgery or unsuitable for custom-made devices or thoracoabdominal off-the-shelf endoprosthesis. ${ }^{6-9}$

The Nellix endovascular aneurysm sealing system (Endologix Inc, Irvine, California), in association with chimney stents (chimney endovascular aneurysm sealing [ChEVAS]), offers an alternative to conventional parallel chimney grafts in combination with EVAR endografts. The newer ChEVAS experience has thus far proven to have low periprocedural risks, comparable to those associated with $\mathrm{f} / \mathrm{bEVAR} .{ }^{10-13}$ The current case report describes a double Nellix ChEVAS procedure in a 77-year-old male patient with an extended symptomatic 66-mm pararenal aortic aneurysm.

\section{Case Report}

A 77-year-old man with a history of type 2 diabetes mellitus, severe chronic obstructive pulmonary disease, and dyslipidemia was submitted to a thoracic-lumbar magnetic resonance imaging in the hospital emergency department, as the patient complained of prolonged (1 month) and persistent back pain. Magnetic resonance imaging revealed an aortic abdominal aneurysm. The patient was swiftly examined by an

\footnotetext{
'Vascular and Endovascular Surgery Department, Sant'Andrea Hospital, University of Rome- “'La Sapienza," Rome, Italy

${ }^{2}$ Anesthesiology Department, Sant'Andrea Hospital, University of Rome"La Sapienza," Rome, Italy

${ }^{3}$ Interventional Radiology Department, Sant'Andrea Hospital, University of Rome-_La Sapienza," Rome, Italy
}

\section{Corresponding Author:}

Roberta Ficarelli, Vascular and Endovascular Surgery Department, Sant'Andrea Hospital, University of Rome-_La Sapienza," Rome 00189, Italy.

Email: robertaficarelli@gmail.com 


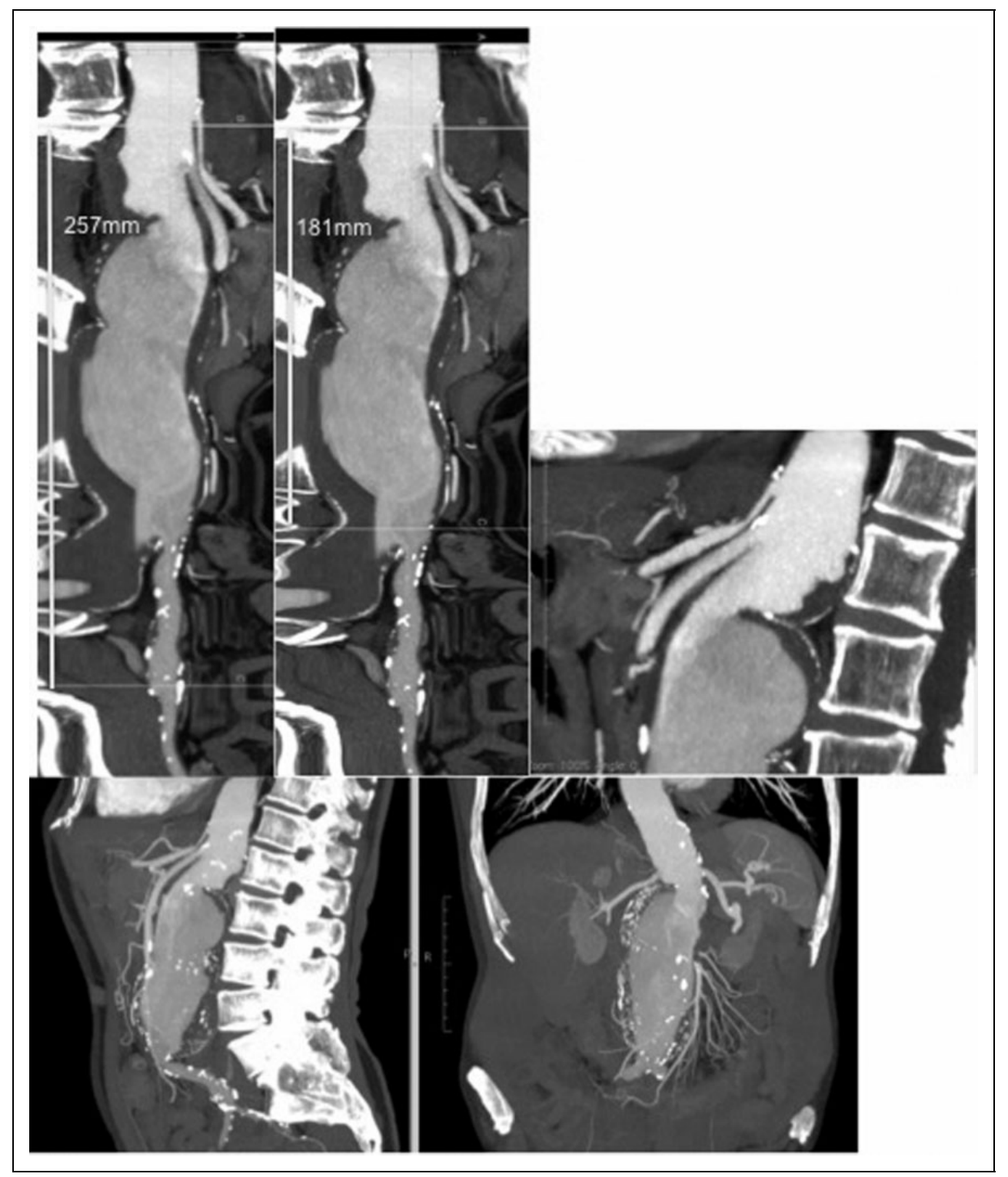

Figure I. Preoperative planning for the chimney endovascular aneurysm sealing (ChEVAS) technique.

angio-computed tomography (angio-CT) scan for a more detailed investigation of the aneurysm.

A 66-mm pararenal aortic aneurysm was diagnosed, associated with extended lymph node disease, a left anterosuperior pulmonary lobe nodule and an infero-ipsilateral disventilatory streak. Because of persistent and increasing back pain, the patient was urgently assessed for a Zenith T-branch stent-graft (Cook Medical, Bloomington, Indiana), but due to inappropriate iliac access ( $6 \mathrm{~mm}$ diameter), the patient was deemed unsuitable. Both common iliac arteries were ectasic, with thrombotic appositions and parietal calcifications. The necessity of entirely cover the common iliac arteries, would have required a conduit through the external iliac arteries but they were of small caliber with parietal calcifications. The patient was also considered unfit for open surgery due to significant comorbidities, the extended lymph node disease, the pulmonary lobe nodule, and the disventilatory streak suggested the presence of a lung disease that could complicate a thoracophrenolaparotomy.

The patient gave informed consent for a double Nellix ChEVAS procedure; chimney grafts deployed into both renal arteries and superior mesenteric artery (SMA), in association with 2 Nellix systems. Preoperative planning identified a proximal tract of the aorta, close to the origin of the almost occluded celiac trunk for the proximal landing zone, and both common iliac arteries, close to the origin of the hypogastric arteries for the distal landing zone (Figure 1). Therefore, a total coverage length of more than $180 \mathrm{~mm}$ called for the deployment of 2 Nellix systems (4 Nellix grafts), bilaterally overlapping (Figure 1).

In an angiographic suite equipped with an image-guided system (GE INNOVA), both common femoral arteries and axillary arteries were accessed percutaneously with the patient 


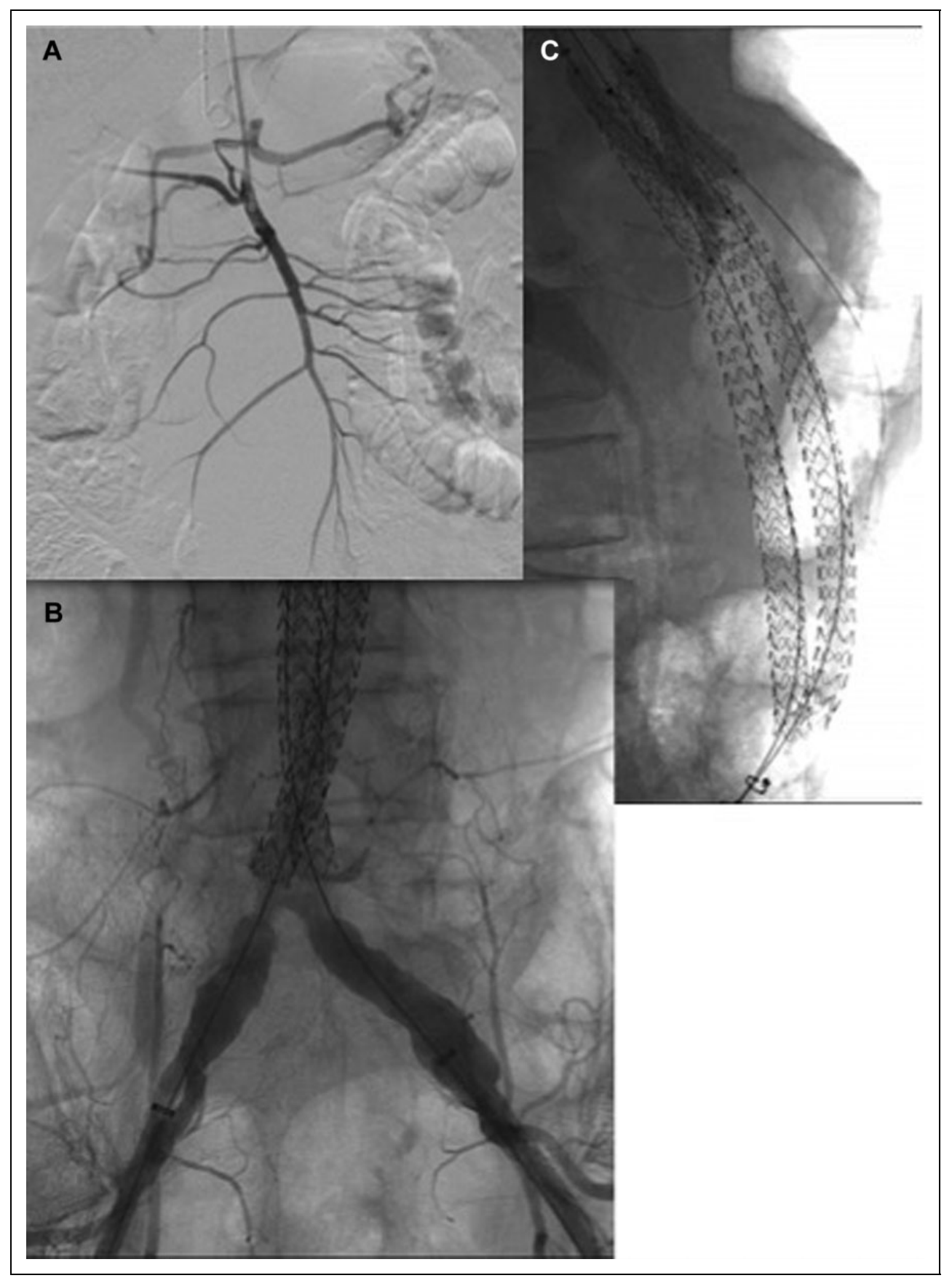

Figure 2. A, Optimal retrograde celiac trunk reperfusion. B, Distal landing zone of the proximal Nellix graft close to the aortic bifurcation. C, Chimney balloons maintained inflated during the entire (pre)filling procedure.

under general anesthesia. Through a short $5 \mathrm{~F}$ sheath, a pigtail catheter was positioned in the descending aorta, and intraoperative angiography evidenced an optimal retrograde celiac trunk reperfusion (Figure $2 \mathrm{~A}$ ).

The visceral arteries were cannulated. One long 7F and two 6F sheaths were advanced through both axillary arteries into the SMA and both renal arteries, respectively, and 3 BeGraft peripheral balloon-expandable covered stents (Bentley InnoMed, Hechingen, Germany) were positioned into the right renal artery $(6 \times 58 \mathrm{~mm})$, the left renal artery $(7 \times 57 \mathrm{~mm})$, and the SMA $(8 \times 37 \mathrm{~mm})$.

The initial 2 proximal $180 \mathrm{~mm}$ Nellix grafts were introduced through the common femoral arteries and positioned with the endobags above the origin of the SMA extending through a distal landing zone close to the aortic bifurcation (Figure 2B). Correct stent position was ensured by intraoperative angiography, and all 3 chimney grafts and the 2 Nellix grafts were simultaneously deployed. The endobags were 


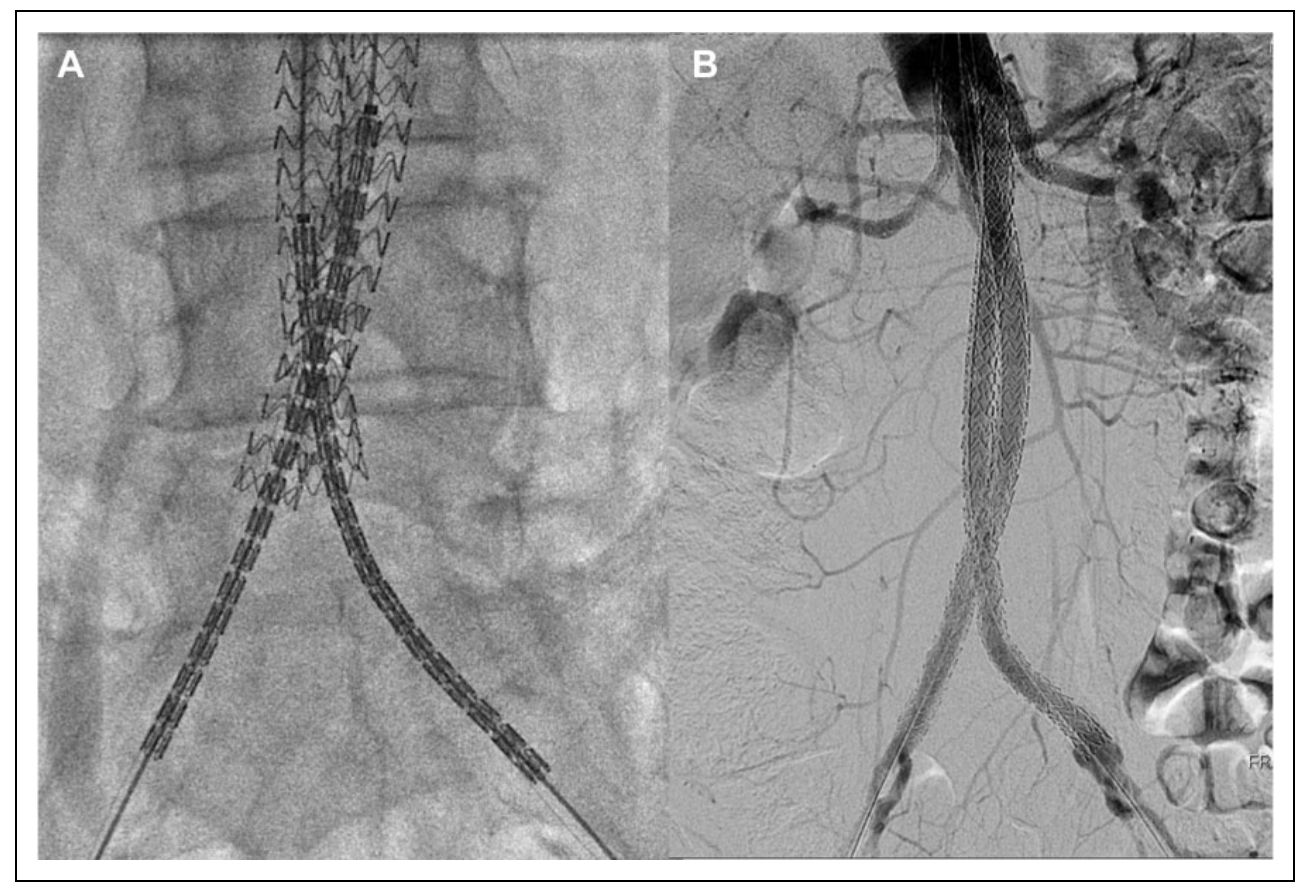

Figure 3. A, The 2 additional Nellix grafts with distal landing zones in both distal common iliac arteries. B, Final angiography illustrating technical success.

prefilled with $190 \mathrm{~mL}$ of a saline solution. Intraoperative angiography showed the absence of proximal gutter endoleak and patency of the chimney grafts. The saline solution was removed and replaced with $190 \mathrm{~mL}$ of the polymer solution up to the recommended pressure of $200 \mathrm{~mm} \mathrm{Hg}$. The balloons of the chimney grafts were maintained inflated during the entire endobag prefilling and filling process (Figure 2C).

The iliac landing zone was determined by intraoperative angiography of the distal aorta. Two additional Nellix grafts $(100 \mathrm{~cm}$ long) were inserted into the uncovered, end portions of the previously deployed Nellix grafts, with an overlap of about 30 $\mathrm{mm}$ and distal landing in both distal common iliac arteries, above the origin of the hypogastric arteries. The Nellix grafts were then inflated with $12 \times 40 \mathrm{~mm}$ balloons, and the process of prefilling the endobags with the saline solution was repeated $(10 \mathrm{~mL})$. The saline solution was replaced with an injection of the polymer solution $(10 \mathrm{~mL})$, and the balloons were deflated (Figure $3 \mathrm{~A}$ ).

Completion angiography evidenced complete exclusion of the aneurysm, patency of the hypogastric arteries, and chimney grafts, with no endoleak, kinking, or compression by the endobag (Figure 3B). The patient was admitted to the intensive care unit for 24 hours, for renal function and liver enzyme monitoring, with blood tests prior to discharge evidencing normal renal function. An angio-CT performed on postoperative day 6 confirmed the completion angiography findings. The patient was discharged on postoperative day 8 , in good general conditions. At 6 months, an angio-CT confirmed ongoing aneurysm exclusion and patency of the aortic and visceral stent grafts with no signs of endoleak, kinking, or migration (Figure 4).

\section{Discussion}

Chimney endovascular aneurysm sealing with the Nellix system is an emerging alternative treatment technique for patients unsuitable for f/bEVAR, with good early results. ${ }^{14}$ However, a long tract of aortoiliac aneurysm involvement (in this case $>180 \mathrm{~mm}$ ) poses a significant technical issue. ${ }^{10-13}$

As reported also by other authors, the initial results of ChEVAS, in challenging cases, unfit for conventional treatments, are encouraging. ${ }^{15}$ Small series of selected cases have been reported in the literature where a suprarenal seal has been obtained with this off-the-shelf solution. ${ }^{14}$

The alternative use of chimney grafts in combination with EVAR endografts for pararenal aneurysm repair has previously been described for patients unsuitable for custom-made devices or open surgery. ${ }^{16-18}$ However, midterm outcomes report relatively high incidences of endoleak and endograft migration. Up to $10 \%$ of chimney procedures performed with standard infrarenal aortic stent grafts have a type 1 Endoleak (EL). ${ }^{9}$ The formation and persistence of "gutters," spaces which form between the endograft and chimney stents, have been correlated with late adverse events. ${ }^{19}$

The Nellix system with endobag sealing, incorporating both the aneurysmal sac and eventual chimney grafts, fills the space where gutters can occur. It is important to maintain the chimney graft balloons inflated during the polymer infusion to avoid excessive compression of the chimney stents.

The choice of optimal chimney graft is also a matter of debate. In the present case, balloon-expandable, covered stents have been used, because the balloon inflation of the graft was 


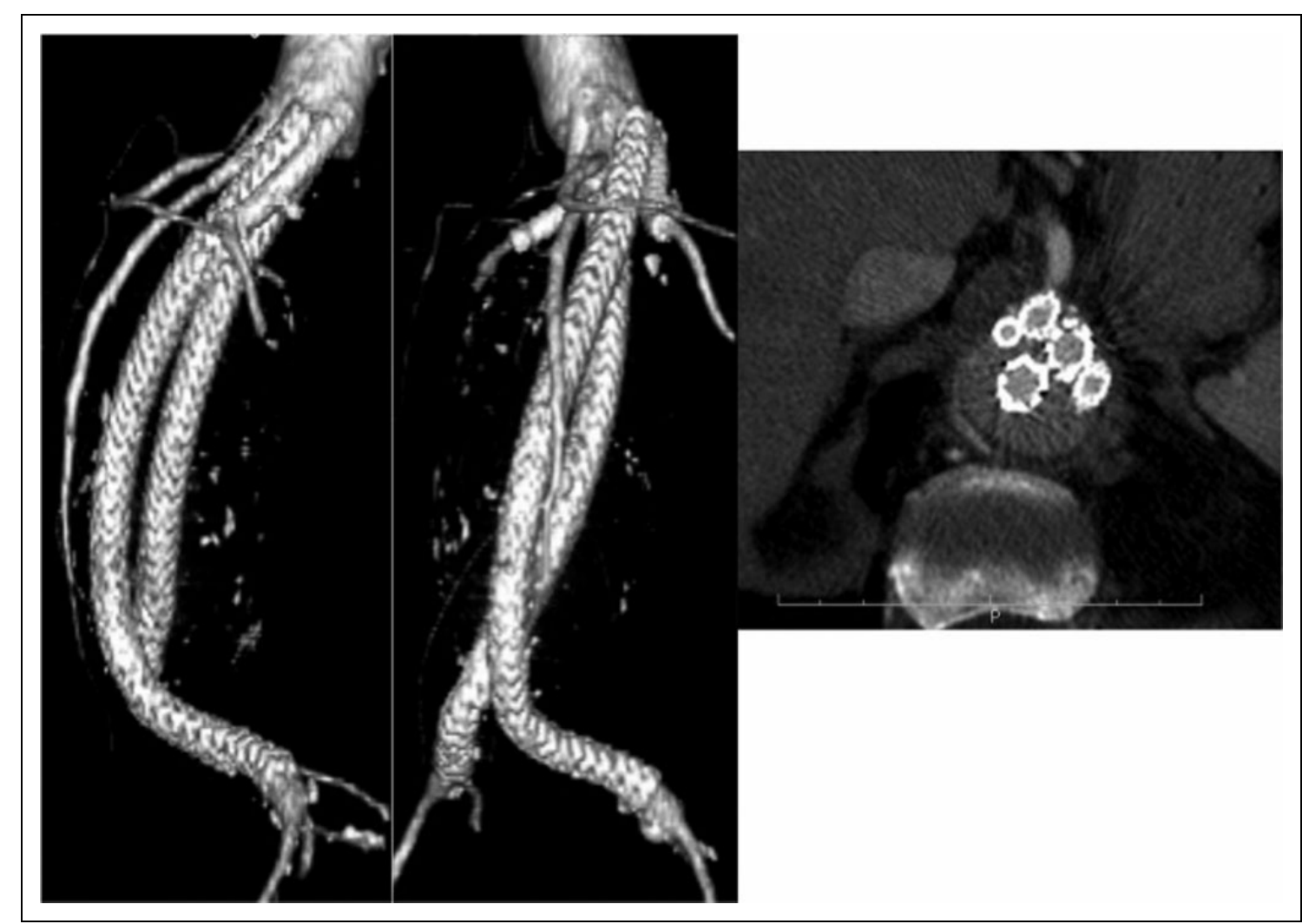

Figure 4. Follow-up at 6 months by angio-computed tomography (CT) confirming aneurysm exclusion and patency of all visceral vessels with endograft integrity in the absence of endoleak.

estimated to minimize possible graft manipulation during the prefilling and filling process of the endobags.

\section{Conclusion}

In this case report, and in very few other reports to date, ${ }^{14}$ the double Nellix ChEVAS solution seems to enable pararenal aneurysm exclusion while preserving visceral vessel patency in patients with an extended pararenal aortic aneurysm unsuitable for open surgery, f/bEVAR, or custom-made alternatives. However, these early results require close follow-up to define whether such techniques could be more extensively applied. ${ }^{15}$

\section{Declaration of Conflicting Interests}

The author(s) declared no potential conflicts of interest with respect to the research, authorship, and/or publication of this article.

\section{Funding}

The author(s) received no financial support for the research, authorship, and/or publication of this article.

\section{References}

1. Verhoeven EL, Vourliotakisa G, Bos WT, et al. Fenestrated stent grafting for short-necked and juxtarenal abdominal aortic aneurysm: an 8-year single-centre experience. Eur J Vasc Endovasc Surg. 2010;39(5):529-536.
2. Greenberg RK, Sternbergh WC III, Makaroun M, et al; Fenestrated Investigators. Intermediate results of a United States multicenter trial of fenestrated endograft repair for juxtarenal abdominal aortic aneurysms. J Vasc Surg. 2009;50(4):730-737.e1.

3. AbuRahma AF, Campbell J, Stone PA, et al. The correlation of aortic neck length to early and late outcomes in endovascular aneurysm repair patients. J Vasc Surg. 2009;50(4):738-748.

4. Leurs LJ, Kievit J, Dagnelie PC, Nelemans PJ, Buth J; EUROSTAR Collaborators. Influence of infrarenal neck length on outcome of endovascular abdominal aortic aneurysm repair. $J$ Endovasc Ther. 2006;13(5):640-648.

5. Brown LC, Greenhalgh RM, Powell JT, Thompson SG; EVAR Trial Participants. Use of baseline factors to predict complications and reinterventions after endovascular repair of abdominal aortic aneurysm. Br J Surg. 2010;97(8):1207-1217.

6. Donas KP, Pecoraro F, Torsello G, et al. Use of covered chimney stents for pararenal aortic pathologies is safe and feasible with excellent patency and low incidence of endoleaks. J Vasc Surg. 2012;55(3):659-665.

7. Moulakakis KG, Mylonas SN, Avgerinos E, et al. The chimney graft technique for preserving visceral vessels during endovascular treatment of aortic pathologies. J Vasc Surg. 2012;55(5): 1497-1503.

8. Tolenaar JL, van Keulen JW, Trimarchi S, Muhs BE, Moll FL, van Herwaarden JA. The chimney graft: a systematic review. Ann Vasc Surg. 2012;26(7):1030-1038. 
9. Katsargyris A, Oikonomou K, Klonaris C, Töpel I, Verhoeven EL. Comparison of outcomes with open, fenestrated, and chimney graft repair of juxtarenal aneurysms: are we ready for a paradigm shift? J Endovasc Ther. 2013;20(2):159-169.

10. Krievins DK, Holden A, Savlovskis J, et al. EVAR using the Nellix sac-anchoring endoprosthesis: treatment of favourable and adverse anatomy. Eur J Vasc Endovasc Surg. 2011;42(1):38-46.

11. Böckler D, Reijnen MM, Krievins D, Peters AS, Hayes P, De Vries JP. Use of the Nellix EVAS system to treat post-EVAR complications and to treat challenging infrarenal necks. $J$ Cardiovasc Surg (Torino). 2014;55(5):601-612.

12. Dijkstra ML, Lardenoye JW, van Oostayen JA, Zeebregts CJ, Reijnen MM. Endovascular aneurysm sealing for juxtarenal aneurysm using the Nellix device and chimney covered stents. $J$ Endovasc Ther. 2014;21(4):541-547.

13. Malkawi AH, de Bruin JL, Loftus IM, Thompson MM. Treatment of a juxtarenal aneurysm with the Nellix endovascular aneurysm sealing system and chimney stent. $J$ Endovasc Ther. 2014;21(4):538-540.

14. Youssef M, Dünschede F, El Beyrouti H, Salem O, Vahl CF, Dorweiler B. Endovascular repair of paravisceral aortic aneurysms combining chimney grafts and the Nellix endovascular aneurysm sealing technology (four-vessel ChEVAS). Thorac Cardiovasc Surg. 2017;65(2):112-119.

15. Torella F, Chan TY, Shaikh U, England A, Fisher RK, McWilliams RG. ChEVAS: combining suprarenal EVAS with chimney technique. Cardiovasc Intervent Radiol. 2015;38(5):1294-1298.

16. Allaqaband S, Jan MF, Bajwa T. "The chimney graft"-a simple technique for endovascular repair of complex juxtarenal abdominal aortic aneurysms in no-option patients. Catheter Cardiovasc Interv. 2010;75(7):1111-1115.

17. Lachat M, Veith FJ, Pfammatter T, et al. Chimney and periscope grafts observed over 2 years after their use to revascularize 169 renovisceral branches in 77 patients with complex aortic aneurysms. J Endovasc Ther. 2013;20(5):597-605.

18. de Bruin JL, Yeung KK, Niepoth WW, et al. Geometric study of various chimney graft configurations in an in vitro juxtarenal aneurysm model. J Endovasc Ther. 2013;20(2):184-190.

19. Coscas R, Kobeiter H, Desgranges P, Becquemin JP. Technical aspects, current indications, and results of chimney grafts for juxtarenal aortic aneurysms. J Vasc Surg. 2011;53(6):1520-1527. 